\title{
Giant lumbar pseudomeningocele after revision lumbar laminectomy: a case report and review of the literature
}

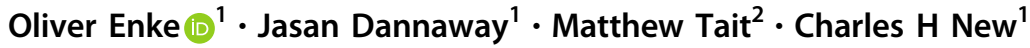

Received: 8 July 2018 / Revised: 14 August 2018 / Accepted: 21 August 2018

(C) International Spinal Cord Society 2018

\begin{abstract}
Introduction Incidental durotomy is a relatively common complication of spine surgery. Prevalence ranges from 3 to $5 \%$ in primary and 7 to $17 \%$ in revision procedures. Despite this relatively common occurrence the subsequent development of pseudomeningoceles following lumbar spine surgery is reported to be between 0.07 and $2 \%$. Giant pseudomeningoceles (GP) are rare and therefore we report our experience with a case.

Case presentation We report a case of an iatrogenic GP post revision lumbar surgery that extended $19 \mathrm{~cm}$ in length. The patient underwent revision L3-S1 laminectomy, laminotomy, excision of pseudomeningocele and successful direct dura repair. Postoperatively, a subcutaneous seroma persisted. This was managed with surgical exploration and prolonged drainage. This led to reduction of the seroma and symptomatic improvement for the patient.

Discussion Incidental durotomy is a relatively common complication of spine surgery and if the durotomy is not addressed at the time of an operation a pseudomeningocele may develop. A pragmatic approach for GP is to individualise management based upon symptoms. Although GP are rare, prevention is likely the best approach. We outlined a structured approach to the management of an incidental dural leak that formed the largest reported pseudomeningocele reported to date.
\end{abstract}

\section{Introduction}

Pseudomeningocele is an extradural accumulation of cerebrospinal fluid (CSF) that extravasates through a dural opening [1]. The aetiology can be traumatic, congenital or iatrogenic [2, 3]. The incidence of pseudomeningoceles following lumbar spine surgery is uncertain, but is reported between $0.07 \%$ [4] and 2\% [5]. Incidental durotomy is more common [6] and it is likely that the incidence of iatrogenic pseudomeningoceles is underestimated. This is because they are often small, self-resolving and may be asymptomatic [7]. However, patients may present with low back

Electronic supplementary material The online version of this article (https://doi.org/10.1038/s41394-018-0118-z) contains supplementary material, which is available to authorized users.

Oliver Enke

dr.oliver.enke@gmail.com

1 Department of Orthopaedic Surgery, Nepean Hospital, Kingswood, NSW, Australia

2 Department of Neurosurgery, Nepean Hospital, Kingswood, NSW, Australia pain, radicular symptoms, persistent headache and local swelling. Giant pseudomeningoceles (GP), defined as over $8 \mathrm{~cm}$ in length [1], are rare, and there is no current consensus on management. We report a case of iatrogenic GP post revision lumbar surgery, which is the largest reported in the literature. We detail the management in order to add to this area of limited evidence.

\section{Case presentation}

A 64-year-old female underwent revision L3-S1 laminectomy and right $\mathrm{L} 4 / 5$ discectomy for radicular pain. Intraoperatively severe lateral canal stenosis at L4/5 and L5/ S1 was decompressed. There was a small incidental durotomy of the nerve root sleeve, adjacent to the right side of L4/5. This was unable to be repaired and was covered with fibrin sealant and absorbable haemostat. One 10Fr Bellovac drain (Co Wellspect, Sweden) was placed in the extradural space and two 10Fr Bellovac drains were left in the subcutaneous layer, the latter two on suction. The patient was mobilised after $36 \mathrm{~h}$ bedrest, routine postsurgical antibiotics (cephazolin) were administered for $48 \mathrm{~h}$ and drains were removed after $48 \mathrm{~h}$. The admission was complicated by poor 
postoperative mobility due to postural dizziness, nausea and headaches, as well as an urinary tract infection requiring oral antibiotics. Over a course of 9 months, the patient developed a large, $20 \mathrm{~cm}$ long bulging fluctuating mass over her surgical site, which was associated with severe local and radiating pain when mobilising and lying flat. Lumbar spine magnetic resonance imaging (MRI) showed a small tear within the posterior dura at level L3, a significant sized collection in the laminectomy defect measuring $2.8 \times 3.1 \mathrm{~cm}$ in transverse diameters and $7 \mathrm{~cm}$ in cephalocaudal length and another long pseudomeningocele in the subcutaneous tissues measuring $19 \mathrm{~cm}$ in length and maximum crosssectional size of $10 \times 5 \mathrm{~cm}$ (Fig. 1). She underwent revision L3-S1 laminectomy, excision of pseudomeningocele and direct dura repair by orthopaedic and neurosurgical teams. The superficial pseudomeningocele drained a large amount of clear CSF, and the membrane was resected thoroughly. Two posterior dural tears were identified and repaired directly using 5-0 monofilament polypropylene sutures (Fig. 2). Intraoperative valsalva manoeuvre demonstrated no further leak. The dural integrity was augmented with application of fibrin sealant followed by drain insertion (10Fr Bellovac drains, 1x extradural and 1x subcutaneous, no suction) and layered closure. Routine postoperative antibiotics (Cephazolin) were administered for $48 \mathrm{~h}$. The patient was kept flat on bedrest for 5 days after which the drains were removed (total output $32 \mathrm{~mL}$ ). The remaining postoperative course was uneventful. At 6 weeks post operatively, re-accumulation of the subcutaneous midline collection was noted and the pseudomeningocele appeared to have recollected. A decision was made to explore surgically, which failed to demonstrate any evidence of a dural defect. On laboratory testing the fluid was negative for Beta 2 Transferrin, hence was not found to be CSF. It is presumed that a large seroma had formed in the previous pseudomeningocele cavity, so the patient was treated with excision of the seroma capsule and prolonged drainage. One extradural and two subcutaneous 14Fr Bellovac drains were left in situ, all on suction. Antibiotics (Clindamycin) were continued for $48 \mathrm{~h}$ post operatively. The deep drain was removed after 5 days and the superficial drains remained for 4 weeks until the total drain output was $<15 \mathrm{~mL}$ in $24 \mathrm{~h}$. All intraoperative cultures remained negative. Serial MRI were obtained every 3 months. At the 6-month follow-up, an MRI demonstrated interval reduction of the subcutaneous seroma spanning from T12 to sacral levels with numerous internal septations, measuring $70 \times 19 \times 63 \mathrm{~mm}$ (Fig. 3). However, there was no palpable collection at the patient's surgical site and she reported improvement in pain and mobility.

\section{Discussion}

Incidental durotomy is a relatively common complication of spine surgery. In primary surgery prevalence ranges from 3 to $5 \%$, and in revision procedures from 7 to $17 \%[6,8]$. If durotomy is not addressed at the time of an operation a

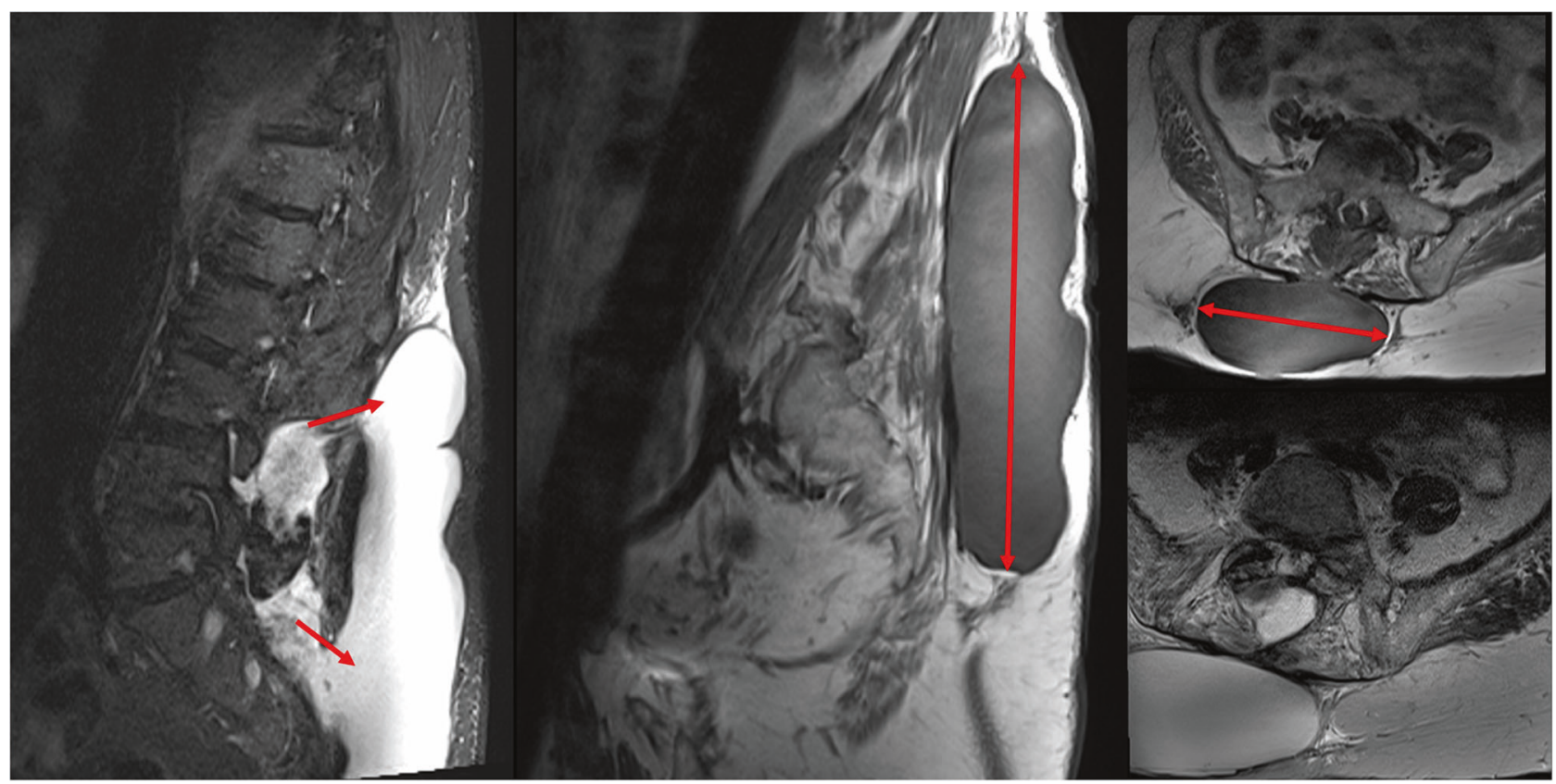

Fig. 1 Preoperative MRI: Collection in laminectomy defect $7 \times 3.8 \times 3.1 \mathrm{~cm}$, decompressing into superficial soft tissues at L2/3 level and mid sacral level. Subcutaneous collection $19 \times 10 \times 5 \mathrm{~cm}$ resembling giant pseudomeningocele 
Fig. 2 Intraoperative images; left: preoperative dura tear in laminectomy defect; right: repair with 5-0 monofilament sutures

Fig. 3 Postoperative MRI (6 months): Reduction in size of posterior subcutaneous seroma $16.3 \times 7 \times 1.9 \mathrm{~cm}$ spanning from T12 to sacral levels, no persisting deep collection
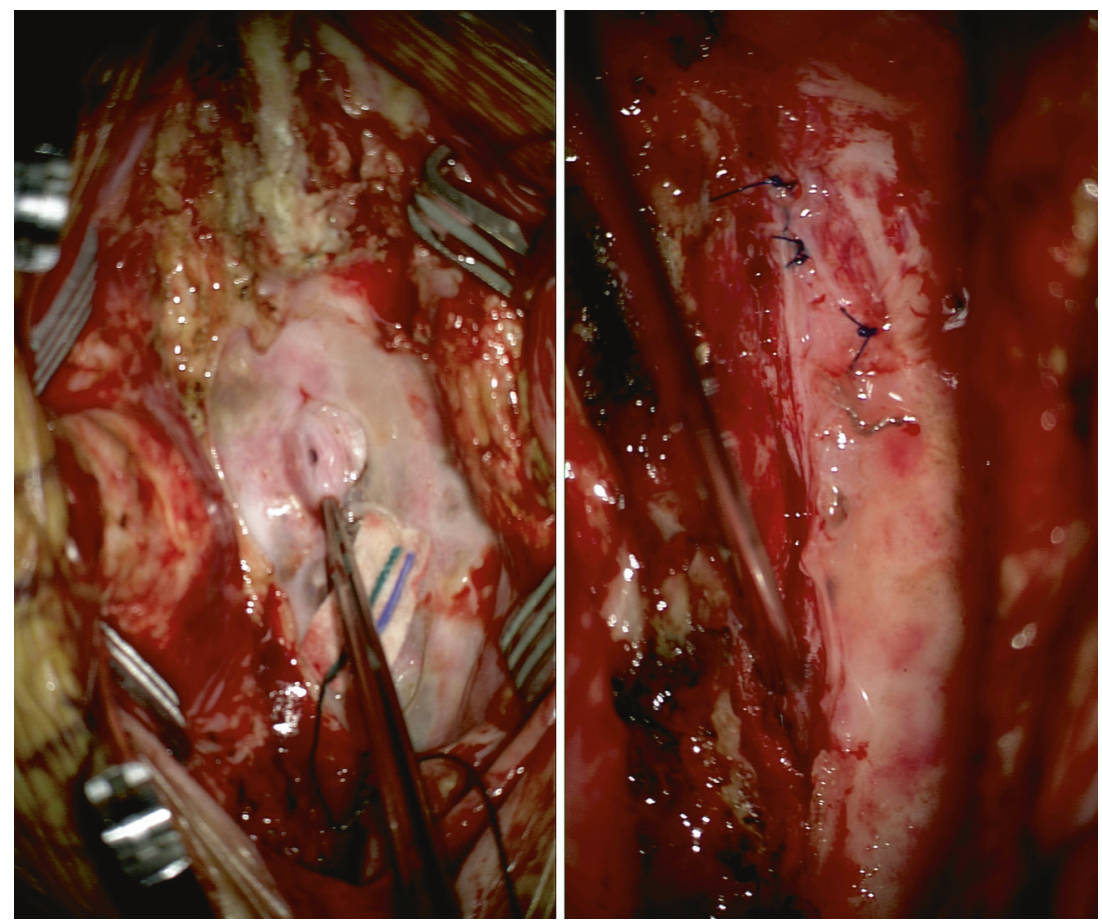

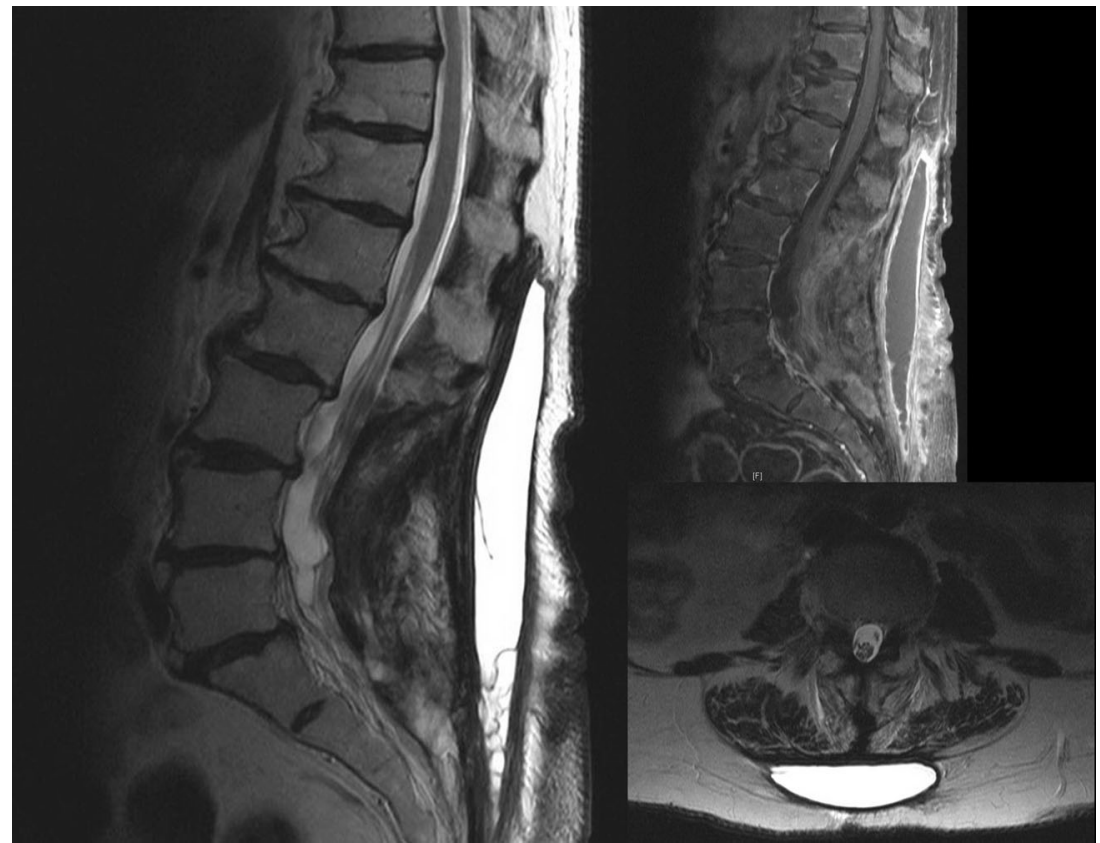

pseudomeningocele may develop, sometimes over years. When there is a tear of both dura and arachnoid layers, CSF can leak into paraspinal tissues. Initially, the fluid may be able to be resorbed, but as a fibrous cystic cavity forms, this does not occur as freely. Eventually a mature pseudocyst wall develops.

Pseudomeningocele may present with back pain, exacerbated by valsalva manoeuvre, headache or swelling. If there is pressure on neural structures, or they are caught in the pseudomeningocele sac, neurological signs and symptoms may be present. MRI is the diagnostic modality of choice as it can characterise, size, contents and location of the pseudomeningocele.

To date, we are aware of 28 reported cases $[9,10]$ of GP. Twenty-one of these cases occurred in the lumbar region and were between 8 and $15 \mathrm{~cm}$ in length. A variety of treatment strategies were implemented, including observation [11], with reported success in all instances. 
Unfortunately, the strength of this evidence is very low, due to high risk of biases.

We feel a pragmatic approach for GP is to individualise management based upon symptoms. Asymptomatic GP can be managed expectantly. If the GP is symptomatic with progressive neurological impairment [1], infection or pain this may be an indication for expedited surgery. The surgical management is controversial, but all techniques are aimed at stemming the egress of CSF by the closure of the durotomy. Options include surgical direct repair, patch techniques (autografts, allografts or fibrin glue), or CSF diversion (lumbar shunt or subarachnoid drain). The techniques used should be tailored to the type, location and size of the dural defect (assessed via imaging and clinical history).

Although GP are rare, prevention is likely the best approach. This involves meticulous surgical technique, active surveillance of incidental durotomy, and primary repair in all instances [8]. A structured approach to the management of incidental durotomy has been outlined, with success reported, in a large series of patients [6], which may serve as a useful approach.

\section{Compliance with ethical standards}

Conflict of interest The authors declare that they have no conflict of nterest.

\section{References}

1. Weng Y-J, Cheng C-C, Li Y-Y, Huang T-J, RW-W. Hsu. Management of giant pseudomeningoceles after spinal surgery. BMC Musculoskelet Disord. 2010;11:53.

2. Hyndman OR, Gerber WF. Spinal extradural cysts, congenital and acquired: report of cases. J Neurosurg. 1946;3:474-86.

3. Cook DA, Heiner JP, Breed AL. Pseudomeningocele following spinal fracture. A case report and review of the literature. Clin Orthop Relat Res. 1989;247:74-9.

4. Swanson HS, Fincher EF. Extradural arachnoidal cysts of traumatic origin. J Neurosurg. 1947;4:530-8.

5. Teplick JG, Peyster RG, Teplick SK, Goodman LR, Haskin ME. CT Identification of postlaminectomy pseudomeningocele. AJR Am J Roentgenol. 1983;140:1203-6.

6. Papavero L, Engler N, Kothe R. Incidental durotomy in spine surgery: first aid in ten steps. Eur Spine J. 2015;24:2077-84.

7. Lee KS, Hardy IM. Postlaminectomy lumbar pseudomeningocele: report of four cases. Neurosurgery. 1992;30:111-4.

8. Guerin P, El Fegoun AB, Obeid I, Gille O, Lelong L, Luc S, et al. Incidental durotomy during spine surgery: incidence, management and complications. A retrospective review. Injury. 2012;43:397-401.

9. Rege SV, Patil H. Iatrogenic lumbar Pseudomeningocele: a case report and review of literature. Rom Neurosurg. 2016;30:429-33.

10. Gupta R, Narayan S. Post-operative Pseudomeningocele after Spine. Surg: Rare Cause Fail Back Syndr IrJNS. 2016;2:15-8.

11. Solomon P, Sekharappa V, Krishnan V, David KS. Spontaneous resolution of postoperative lumbar pseudomeningoceles: A report of four cases. Indian J Orthop. 2013;47:417-21. 